\title{
Exciton induced photodesorption in rare gas solids
}

\author{
Takato Hirayama $^{1}$ and Ichiro Arakawa ${ }^{2}$ \\ ${ }^{1}$ Department of Physics, Rikkyo University, Nishi-Ikebukuro, Toshimaku, Tokyo \\ 171-8501 JAPAN \\ ${ }^{2}$ Department of Physics, Gakushuin University, Mejiro, Toshimaku, Tokyo \\ 171-8588 JAPAN \\ E-mail: hirayama@rikkyo.ac.jp, ich.arakawa@gakushuin.ac.jp
}

\begin{abstract}
This paper reviews our progress on the desorption induced by electronic transitions (DIET) in rare gas solids by selective excitation of valence excitons. Observation of metastable atoms desorbed by excitonic excitation gives us direct information on the exciton-induced desorption processes in rare gas solids. Validity of three desorption mechanisms, cavity ejection, excimer dissociation, and internal sputtering, are demonstrated by systematic measurements of kinetic energies and angular distributions of desorbed particles. Absolute yield of total and partial desorption was measured, which can lead us to the quantitative understanding of exciton-induced desorption processes.
\end{abstract}

Submitted to: J. Phys.: Condens. Matter

\section{Introduction}

Desorption of atoms from the surface of solid rare gases (RGS) has been extensively studied in the past two decades (Zimmerer 1994, Arakawa 1998). RGS is a model system to investigate the dynamic processes of desorption induced by electronic transitions (DIET) because of its simplicity and similarity of the electronic structure to the isolated atoms.

The correlation between exciton creation and atomic desorption in RGS was first suggested by the electron stimulated desorption (ESD) experiment for solid Ar (Coletti et al 1984). Feulner et al (Feulner et al 1987) clearly demonstrated that the desorption of atoms can be initiated by the creation of surface and bulk excitons for monolayers and multilayers of $\mathrm{Ar}$ and $\mathrm{Kr}$ by state-selective excitation with synchrotron radiation. Further breakthrough was achieved by selective detection of desorbed species (Kloiber et al 1988, Kloiber \& Zimmerer 1989) by combining massspectroscopic and luminescence technique. They measured partial desorption yields of excited atoms in $n \mathrm{p}^{5}(n+1) \mathrm{s}\left({ }^{3} \mathrm{P}_{1}\right.$ and $\left.{ }^{1} \mathrm{P}_{1}\right), n \mathrm{p}^{5}(n+1) \mathrm{s}\left({ }^{3} \mathrm{P}_{0,2}\right)$, and $n \mathrm{p}^{5}(n+1) \mathrm{p}$ states ( $n=2$ for Ne, $n=3$ for Ar) separately from the total yield.

Detailed information on the exciton-induced desorption processes was also provided by low energy electron impact experiments (Arakawa et al 1989, Weibel et al 1993, Leclerc et al 1990, Leclerc et al 1992). They discussed the desorption mechanism using the results on the kinetic energy and angular distribution of desorbed metastable atoms. Another important contribution to this field was ion-impact 
experiments (Ellegaard et al 1986, Schou 1987, Johnson \& Brown 1982, Reimann et al 1984, Reimann et al 1988). They clearly demonstrated the significance of the exciton-induced desorption even in the ion-induced sputtering phenomena (Johnson \& Inokuti 1983).

For the desorption induced by exciton creation from the surface of solid rare gases, excimer dissociation (ED) and cavity ejection (CE) mechanisms were proposed (Coletti et al 1984, Coletti \& Debever 1984) and have been confirmed experimentally. The desorption via ED process is due to a dissociation of a molecular type exciton similar to the dissociation of an excited dimer (excimer) in the gas phase. Potential curves relevant to the ED process is schematically shown in figure 1. Initial excitation (A) forms a vibrationally excited excimer. During or after the vibrational relaxation (B), the excimer can decay to the ground state (C), which leads to the desorption of a ground state atom. Excitation to higher level (A') can results in the desorption of an atom in excited state (D').

Figure 2 shows the cavity ejection mechanism schematically. Negative electron affinity (or positive $V_{0}$ value) of the matrix is known to be essential for the desorption of an excited atom via $\mathrm{CE}$ mechanism to have a repulsive interaction between the excited atom and the surrounding ground state atoms. While all rare gases in gas phase have negative electron affinity, the sign changes to positive for solid $\mathrm{Kr}$ and solid Xe because of their large polarizability (Schwentner et al 1975). Systematic measurements (Runne et al 1993) revealed a clear correlation between the sign of the electron affinity and the desorption via CE mechanism. This mechanism has been discussed by molecular dynamics calculation for solid Ne (Dutkiewicz et al 1996) and solid Ar (Cui \& Johnson 1989). The calculated results for the kinetic energies of desorbed excited atoms were in good agreement with the experimental results. It should be worth noting that desorbed atoms via CE process are essentially in excited states.

Internal sputtering (IS) mechanism is another process for the desorption of excited and ground state atoms. In ED process, dissociation of an excimer release the kinetic energy in the order of $1 \mathrm{eV}$. If ED occurs in the vicinity of the surface, this large energy release may blow off many atoms in/near the surface layer, which can significantly contribute to the desorption. IS process has been quantitatively discussed by a molecular dynamics calculation for solid Ar (Cui et al 1988) and experimentally observed by our group (Arakawa et al 1995). IS process can be also possible in the desorption via CE mechanism (Dutkiewicz et al 1996). An excited atom created just below the surface may be ejected into the vacuum via CE mechanism, thereby pushing aside the atoms in the overlayer, which may also desorb due to the small cohesive energy of rare gas solid.

Here, we present our results on the photo-desorption experiments from the surface of solid rare gases, mainly solid Ne, by selective creation of valence excitons by synchrotron radiation. Validity of the desorption mechanisms described above will be discussed with our experimental results on the excited atom desorption. We also show the absolute desorption yields, which can lead us to the quantitative understanding of the exciton induced desorption processes. 


\section{Experimental}

\subsection{Beam line}

Experiments presented here were performed using the beam line BL5B, UVSOR Facility of the Institute for Molecular Science, Okazaki. Detail of the beam line is described elsewhere (Sakurai et al 1989, Sakurai et al 1990). A plane grating monochromator (PGM) covers a wavelength range of 2 to $240 \mathrm{~nm}$ by a combination of three gratings $\left(\mathrm{G}_{1}-\mathrm{G}_{3}\right)$ and 7 post mirrors $\left(\mathrm{M}_{2}^{0}-\mathrm{M}_{2}^{6}\right)$. The wavelength resolution $(\lambda / \Delta \lambda)$ at the region of excitonic excitation in rare gas solids is 100 to 500 depending on the width of the exit slit.

A photon beam monitor was used for the measurements of absolute number of photons incident on the sample surface (Hirayama et al 1997). It consists of a beam defining aperture of $3 \mathrm{~mm}$ in diameter and a thick Au sheet. Negative voltage $(-45 \mathrm{~V})$ was applied to the Au sheet by a dry battery. The number of photons was estimated from the photoemission current emitted from the Au surface and the photoelectric yield (number of electrons per incident photon) of Au reported by Samson (Samson 1964), and was typically in the order of $10^{9} \mathrm{~s}^{-1}$.

\subsection{Main chamber}

Schematic of the main chamber is shown in figure 3. The chamber was evacuated by a turbo molecular pump and a Ti-sublimation pump, and the base pressure was about $5 \times 10^{-9} \mathrm{~Pa}$.

The sample film was prepared on a $\operatorname{Pt}(111)$ disk of $8 \mathrm{~mm}$ diameter. This was fixed to a liquid He cryostat and cooled down to about $6 \mathrm{~K}$. The cryostat was surrounded by a liquid $\mathrm{N}_{2}$-cooled heat shield. The sample film was condensed on the Pt disk by filling the chamber with a sample gas to a pressure of $10^{-6} \sim 10^{-4} \mathrm{~Pa}$. The film thickness was estimated from the exposure assuming the condensation coefficient to be unity.

\subsection{Time-of-flight measurements}

For the measurements of the time-of-flight spectra of desorbed metastable atoms, the pulsed incident light must be used. Since the kinetic energy of desorbed species does not exceed a few eV, the flight-time is in the order of $10^{-5} \sim 10^{-3} \mathrm{~s}$, which is much longer than the interval of synchrotron radiation pulses from the storage ring even in single bunch operation. Therefore we have used a mechanical chopper for pulsing the incident light. A disc of $40 \mathrm{~cm}$ in diameter was mounted and driven by a DC motor via a rotational feedthrough. The FWHM and the frequency of the photon pulse were typically $15 \mu$ s and $400 \mathrm{~Hz}$, respectively.

Detected metastable atoms were in $n \mathrm{p}^{5}(n+1) \mathrm{s}\left({ }^{3} \mathrm{P}_{0,2}\right)$ state, which have lifetimes much longer (in Ne case, $430 \mathrm{~s}$ for $J=0,24.4 \mathrm{~s}$ for $J=2$ (Small-Warren \& Chiu 1975)) than the flight time between the sample and the detector $(10 \mu \mathrm{s} \sim 1 \mathrm{~ms})$.

\subsection{Absolute desorption yield measurements}

2.4.1. Metastable atom desorption Absolute yield of metastable atom desorption was determined by measuring the intensity of the desorbed metastable atoms, number of incident photons, geometrical conditions, and the angular distribution of the 
desorption (Hirayama et al 1997). Desorbed metastable atoms are detected by an open electron multiplier tube (EMT, Hamamatsu, R595) with a CuBe dynode as the first electrode. The EMT was fixed at a distance of $360 \mathrm{~mm}$ from the sample in the normal direction of the sample. The diameter of the entrance of the EMT is $8 \mathrm{~mm}$ which corresponds to the detection solid angle of $3.1 \times 10^{-5} \mathrm{sr}$.

The absolute desorption yield, $Y_{\mathrm{M}}$ was calculated using the following equations,

$$
\begin{aligned}
& Y_{\mathrm{M}}=N_{\mathrm{M}} \times F\left(\Omega_{\mathrm{d}}, n\right) \\
& N_{\mathrm{M}}=\frac{C_{\mathrm{M}}}{\eta_{\mathrm{EMT}}} \times \frac{1}{N_{\mathrm{ph}}}
\end{aligned}
$$

where $N_{\mathrm{M}}$ and $C_{\mathrm{M}}$ are the absolute yield detected by EMT per photon and detected count rate of the metastable atoms, respectively, $\eta_{\mathrm{EMT}}$ is the detection efficiency of EMT for metastable atoms, $F\left(\Omega_{\mathrm{d}}, n\right)$ is a factor which takes into account the geometrical condition and the angular distribution of the desorbed atoms, and is calculated as follows,

$$
F\left(\Omega_{\mathrm{d}}, n\right)=\frac{\int_{0}^{2 \pi} \int_{0}^{\frac{\pi}{2}} \cos ^{n} \theta d \theta d \phi}{\int_{\Omega_{\mathrm{d}}} \cos ^{n} \theta d \Omega}
$$

where $\Omega_{\mathrm{d}}, \theta, \phi$, and $\cos ^{n} \theta$ are the solid angle of the detector, the polar angle, the azimuth angle, and the fitting function for the angular distribution of the desorbed atoms, respectively.

The quantum efficiency of EMT for metastable atoms has been reported by several authors (Borst 1971, Alvariño et al 1984) to be 0.12 and 0.035 for $\mathrm{Ne}$ and Ar, respectively. The angular distributions of the desorbed metastable atoms from solid Ne by exciton creation have already been reported by our group (Arakawa et al 1995, Sakurai et al 1995, Weibel et al 1993).

2.4.2. Total desorption Absolute yield of total desorption from the surface of solid rare gas was estimated from the partial pressure change in the main chamber during the irradiation of light, number of incident light, and the pumping speed of the pumping system for the desorbed gas (Arakawa et al 2000, Arakawa et al 2003, Adachi et al 2003).

The desorption rate was calculated from the pumping speed for corresponding gas and the rise of the partial pressure in a vacuum chamber during irradiation of the sample. The pumping speed of a turbomolecular pump and cold surfaces was $0.14 \pm$ $0.01 \mathrm{~m}^{3} / \mathrm{s}$ in total for $\mathrm{Ne}$, which was determined from the Ne pressure measured by an extractor gauge installed in the main chamber and from the flow rate calibrated volumetrically using a reference volume and a Baratron pressure gauge as reference. The small rise of the partial pressure during irradiation was detected by a quadrupole mass spectrometer which was calibrated against the extractor gauge for each run of the experiment. It should be noted that the uncertainty of the relative sensitivity of the extractor gauge for each gas was cancelled in the present method for determining the desorption rate. 


\section{Experimental Results and Discussion}

\subsection{Excited atom desorption}

3.1.1. Wavelength dependence of metastable desorption yield. Figure 4 shows desorption yield of the metastable atoms in ${ }^{3} \mathrm{P}_{0,2}$ states via the cavity ejection mechanism from the surface of solid $\mathrm{Ne}$ as a function of the wavelength of the incident light for sample thicknesses of (a) $500 \mathrm{ML}$ and (b) 5 ML. Observed peaks were assigned to a series of bulk (B) and surface (S) excitons (Saile \& Koch 1979). The close correlation between exciton creation and metastable desorption was clearly demonstrated. It should be noted that only the surface peaks (S1 and S') are observed at the thin sample.

3.1.2. Time-of-flight spectra of desorbed metastable atoms. Figure 5 shows time-offlight spectra of desorbed metastable atoms from the surface of solid Ne measured at the wavelengths of $72.3 \mathrm{~nm}, 70.7 \mathrm{~nm}, 65.4 \mathrm{~nm}$ and $61.1 \mathrm{~nm}$, which correspond to the excitation energies of the 1st order surface (S1) and bulk(B1) excitons, $2 \mathrm{p}^{5} 3 \mathrm{p}$-type surface exciton (S') and the 2nd order bulk exciton (B2), respectively.

The strongest peak at flight time $t_{\mathrm{f}}=0$ is due to scattered and emitted light from the sample. The higher kinetic energy peak $\left(t_{\mathrm{f}} \sim 100 \mu \mathrm{s}, E_{\mathrm{k}}=1.4 \pm 0.1 \mathrm{eV}\right)$ is due to metastable Ne atoms desorbed via the excimer dissociation (ED) process. ED peak was observed in the TOF spectra by $\mathrm{S}^{\prime}$ and B2 excitation but not in S1 and B1 excitation. This is because only ground state atoms, which can not be detected in the present measurements, desorbed by the excitation of the 1st order excitons.

The peak at $t_{\mathrm{f}} \sim 250 \mu \mathrm{s}$ is due to the cavity ejection (CE) mechanism. In the CE scheme, the motive force of the desorption of excited atom is the repulsive interaction between the excited atom and the surrounding ground state atoms, therefore, the kinetic energy of desorbed excited atoms should depend on the electronic state of the excited atom. The kinetic energies of CE peaks for S1, B1, and B2 excitation are found to be the same within the experimental uncertainty $\left(E_{\mathrm{k}}=0.18 \pm 0.02 \mathrm{eV}\right)$. This fact suggests that the desorbed $\mathrm{Ne}^{*}(\mathrm{CE})$ at $\mathrm{S} 1, \mathrm{~B} 1$, and $\mathrm{B} 2$ excitation are, at least when leaving from the surface, all in the same electronic states, probably in $2 \mathrm{p}^{5} 3 \mathrm{~s}\left({ }^{3} \mathrm{P}_{0,2}\right)$. The initial electronic state of $\mathrm{B} 2$ exciton $\left(2 \mathrm{p}^{5} 4 \mathrm{~s}\right)$ is known to relax very rapidly $\left(\sim 10^{-13} \mathrm{~s}\right)$ to the 1 st order excitonic state (Schwentner \& Koch 1976), which is consistent with the discussion above. Additional shoulder appeared in the higher energy side in B2 spectrum $\left(t_{\mathrm{f}} \sim 180 \mu \mathrm{s}\right)$, whose kinetic energy is $0.36 \pm 0.04 \mathrm{eV}$, can be the contribution of $\mathrm{Ne}^{*}$ in $2 \mathrm{p}^{5} 4$ s state at the desorption.

In the TOF spectra (figure 5), a tail was observed by the bulk excitation (B1 and B2). Figure 6 shows the wavelength dependence of the intensity of CE peak (solid line) and the area of the tail (open circles) $\left(t_{\mathrm{f}}: 600 \sim 900 \mu \mathrm{s}, E_{\mathrm{k}}: 0.01 \sim 0.03 \mathrm{eV}\right.$ ). It is clearly seen that this tail appeared only by the bulk excitation. This tail can be attributed to desorption via IS mechanism, i.e. an excited atom created just below the surface has lost its energy by blowing off the atoms in the overlayer before desorption via CE process. Low kinetic energy and broad angular distribution (see section 3.1.3 below and table 2) support this idea. Our result is in consistent with a molecular dynamics simulation (Dutkiewicz et al 1996). The simulation shows that an exciton created in 5 outermost layers can desorb via CE mechanism with a kinetic energy lower than $0.1 \mathrm{eV}$.

Table 1 summarize the kinetic energies of desorbed excited atoms by S1, B1, S', 
and B2 excitation for solid Ne together with their desorption mechanism and electronic state at the desorption.

3.1.3. Angular distribution of desorption. Motive force of the desorption via the cavity ejection mechanism is the repulsive force from ground state atoms surrounding the excited atom, which should be axially symmetric around the sample normal direction (see figure 2). The desorbed excited atoms, therefore, should have a sharp angular distribution towards the sample normal direction. Figure 7 shows the results of the angular distribution of the CE peak of desorbed metastable Ne atoms by S1 excitation (Arakawa et al 1995). Very sharp distribution was observed as expected: $n=14$ if the distribution is fitted to $\cos ^{n} \theta$. The sharpness varied with the excitation energy, i.e., the kind of exciton created, as summarized in table 2. Such a narrow distribution is explained qualitatively in terms of the vibrational displacement of $\mathrm{Ne}$ atoms at excitation by simple trajectory calculations (Sakurai et al 1995). This is also supported by the recent ESD measurements where FWHM of the angular distribution increases as the sample temperature rises (Kato et al 2006).

The ED component by S' excitation showed a much broader angular distribution $(0 \leq n \leq 1$, FWHM $\geq 120$ deg.). Molecular dynamics calculation for solid $\mathrm{Kr}$ (Dutkiewicz et al 1995) showed that an excimer formed in the surface layer easily "forget" their initial orientation along crystal axes before dissociation, which may explain the broad angular distribution in ED component. Another possible explanation is that the excimer created in the surface layer desorbs and dissociates in vacuum (see section 3.2), resulting in the isotropic angular distribution.

Throughout the series of angular distribution measurements of the desorbed $\mathrm{Ne}^{*}$, no azimuthal structure around the sample normal was observed for any TOF components, as was the case for ESD Ar* (Leclerc et al 1992).

3.1.4. Desorption by $2 p^{5} 3 p$-type surface exciton. Excited atom desorption through the creation of S' exciton can be a good test for the validity of the cavity ejection mechanism. Electronic configuration of $\mathrm{S}^{\prime}$ exciton is $2 \mathrm{p}^{5} 3 \mathrm{p}$, which is optically forbidden state but partly allowed at the surface and the lattice defect due to reduced symmetry (Inoue et al 1984). This configuration has 10 states, whose excitation energies in gas phase is from 18.382 to $18.966 \mathrm{eV}$ (Saloman \& Sansonetti 2004). Kloiber and Zimmerer demonstrated that excited atoms in these states desorb by S' exciton creation (Kloiber \& Zimmerer 1990).

Figure 8(a) shows a series of time-of-flight (TOF) spectra of metastable atoms in $2 \mathrm{p}^{5} 3 \mathrm{~s}\left({ }^{3} \mathrm{P}_{0,2}\right)$ states desorbed via $\mathrm{CE}$ process from the surface of solid Ne, and figure 8 (b) shows a dependence of $\mathrm{Ne}^{*}$ desorption yield on the wavelength of the incident light in the energy range corresponding to the excitation of $2 \mathrm{p}^{5} 3 \mathrm{p}$-type surface exciton (S'). Thickness of the sample was $690 \mathrm{ML}$. The wavelengths where each TOF spectrum was measured are marked by open circles in figure 8(b). It is clearly seen from the figure that there are 2 components in TOF spectra, one whose kinetic energy increases with the energy of incident light (peak A), and the other whose kinetic energy is fixed (peak B). Figure 9 shows the kinetic energy spectra converted from figure 8(a). Dots show the measured points and solid lines are fitted curves using 2 Gaussian functions. The kinetic energy of the peak $\mathrm{A}$ as a function of the incident photon energy is plotted in figure 11. The kinetic energy increases linearly with increasing the incident photon energy, i.e., the desorbed atom in higher electronic state gains larger kinetic 
energy, which supports the validity of the cavity ejection mechanism. The thickness dependence of the TOF spectra (figure 10) shows that the peak A is the contribution of the excitation of S' exciton at the surface, while the peak B is due to the bulk excitation, probably at the lattice defect, which may explain why the kinetic energy of the peak B does not depend on the incident wavelength.

\subsection{Excimer desorption}

Desorption of excited dimers from the surface of rare gas solids was first suggested by Coletti et al (Coletti et al 1984) for solid Ar irradiated by low energy $(\leq 100$ $\mathrm{eV}$ ) electron beam. Reimann and co-workers (Reimann et al 1988, Reimann et al 1990, Reimann et al 1992) systematically studied the sputtering and luminescence at the surface of solid Ar caused by $\mathrm{MeV}$ light ions. They detected the luminescence of the desorbed excimers by observing only the plume in front of the Ar sample using such an experimental geometry that the emission from the sample was blocked by the edge of the sample substrate. From the detailed analysis of the results, they concluded that $\mathrm{Ar}_{2}{ }^{*}{ }^{3} \Sigma_{u} ; 1_{u}, 0_{u}^{-}$desorbed with a kinetic energy around $0.1 \mathrm{eV}$, and that many-body collisions during the dimerization and desorption efficiently relaxed the vibrational levels of the excimers. Excimer desorption by low energy photon was first reported by Grigorashchenko et al (Grigorashchenko et al 1997). for solid Ar and by Savchenko et al. (Savchenko et al 1997) for solid Ne. Their results suggested that the desorption of excited dimers was closely related to the exciton creation.

Molecular dynamics calculations by Johnson and co-workers have shown that the desorption of excited dimers was energetically possible for $\mathrm{Ar}_{2}{ }^{*}$ in ${ }^{1,3} \Sigma_{u}$ states (Cui et al 1989) and for $\mathrm{Kr}_{2}{ }^{*}$ in ${ }^{1} \Sigma_{u}$ state (Buller \& Johnson 1991). They pointed out that the excimer desorption was related to the formation of a 'cavity' around a moleculartype self-trapped exciton like in the atomic desorption case. Chen et al. (Chen et al 1996) have studied the desorption of an excimer from solid Ne using the extended-ion method on the basis of one-electron Hartree-Fock approximation. Their results also supported the cavity ejection mechanism in the case of excimer desorption.

We have observed the desorption of excimers $\mathrm{Ne}_{2}{ }^{*}$ in ${ }^{3} \Sigma_{u}$ state by observing a luminescent 'plume' in front of the sample surface by low energy $(20-200 \mathrm{eV})$ electron impact and irradiation of $55-75 \mathrm{~nm}$ synchrotron radiation (Hirayama et al 2001). Figure 12(a) shows the dependence of the excimer desorption yield, or the plume emission intensity, on the wavelength of the incident light. Also shown in 12(b) is the desorption yield of excited Ne atoms via the cavity ejection mechanism. The agreement in peak positions between the $\mathrm{Ne}^{*}$ desorption yield and the plume intensity shows that the plume emission is closely related to the valence exciton creation.

The average kinetic energy of desorbed $\mathrm{Ne}_{2}{ }^{*}$ was estimated at $0.2 \pm 0.1 \mathrm{eV}$ by analyzing the shape of the emission plume. This estimation is in fair agreement with Chen et al.'s value $0.23 \mathrm{eV}$ (Chen et al 1996) theoretically obtained for $\mathrm{Ne}_{2}{ }^{*}$ desorbed from (100) face of solid Ne. These low values of kinetic energy suggest that the cavity ejection model is also plausible for the desorption of excimers.

The decay of the plume emission was not of single exponential type, owing to the dependence of the emission lifetime on the vibrational level of the excimers (Cohen \& Schneider 1974). Detailed analysis showed that most of the desorbed excimers were in the highest vibrational level, suggesting that the desorption process is much faster than vibrational relaxation. 


\subsection{Absolute desorption yields}

3.3.1. Total desorption For the quantitative understanding of the mechanism for exciton induced desorption and relaxation processes, we have measured absolute desorption yields in the excitonic excitation region. Total desorption yield from the surface of solid Ne in the excitonic excitation range is shown in figure 13 (Arakawa et al 2000). High background yield is due to the second order light of the monochromator, fraction of which was measured to be $20 \sim 35 \%$ in this wavelength region (Sakurai et al 2002). Peaks by the excitation of the surface excitons ( $\mathrm{S} 1$ and $\mathrm{S}^{\prime}$ ) are clearly visible in the spectrum of the thickness of $73 \mathrm{ML}$ in addition to those by the bulk excitons (B1, B2 and B3). At the excitation energy higher than the band gap energy, a rise of yield was observed in the results of thicker sample. At this energy, an ion can be created, which may lead to the formation of either an exciton or an excited dimer (excimer) via an electron-hole recombination. They result in the cavity ejection and the excimer dissociation processes, respectively. The latter, the formation of the excimer followed by the dissociation, can be the dominant process for the total desorption after ionization.

The thickness dependences of the total desorption yields at the excitation of S1, B1, S' and B2 excitons are shown in figure 14. The yields were estimated from the peak height above the continuous background. Contribution of the 2 nd and 3rd order light was taken into account. For the films thinner than 20 atomic layers, it was difficult to determine the yields because of high background signal. The yields by the bulk excitation, B1 and B2, increase as the film becomes thicker, and seem to reach constant values, $1.6 \pm 0.3$ and $1 \pm 0.2$ (atoms/photon), respectively, at the thickness around $100 \sim 200$ ML. Absolute yields by the excitation of surface excitons, S1 and S', on the other hand, showed no dependence on the film thickness, as is expected for surface excitations.

One can obtain the ratio of the number of desorbed atoms to that of surface excitons created by photons by the absolute value of the total desorption yield. Number of surface excitons created can be estimated by the absorption coefficient of solid $\mathrm{Ne}$ at the wavelength corresponding to the bulk exciton (B1) excitation. We assume that the excitation probability of S1 is the same as that of B1 in each layer at their excitation energy. The estimation based on the photoabsorption data (Pudewill et al 1976) leads to 0.1 excitons per photon. It is likely that a surface exciton can desorb at most a single atom, the excited atom itself in the CE process. Although this estimation, 0.1 excitons per photon, is considerably smaller than the observed yields, 0.3 atoms per photon by the creation of $\mathrm{S} 1$ exciton, we concluded that almost all the surface exciton yield the desorption of one Ne atom or, in other words, that the desorption probability of the surface exciton S1 is almost unity. The larger desorption yield in comparison with the estimated efficiency for an exciton creation may be explained by other desorption process: the CE process of a dimer and the ED process which yield two or more desorbing atoms. Another possibility is a contribution of reflected light on the sample substrate, which may enhance the number of excitons on the surface.

Similar measurements were performed for solid Kr (Adachi et al 2003). The thickness dependence of the yields for B1(3/2) exciton is shown in figure 15 . Solid lines are the results of a simulation. In this simulation we assumed that the desorption by a bulk exciton creation is caused by the sequential processes as i) diffusion of the exciton, ii) formation of an excimer, iii) dissociation of the excimer, and iv) 
collision cascade followed by internal sputtering. The initial distribution of bulk excitons was estimated from the photoabsorption coefficient for solid $\mathrm{Kr}$ (Haensel et al 1970, Skibowski 1971, Sonntag 1977), and a classical molecular dynamics calculation (Dutkiewicz et al 1995) was used for the estimation of collision cascade. Diffusion length $L$ for excitons was the single adjustable parameter in this simulation. As shown in the figure, our experimental results, both absolute values and thickness dependence, were well reproduced by the diffusion length $L$ between $5 \sim 10 \mathrm{~nm}$. Because the diffusion length may strongly depend on the crystalline condition and the sample temperature, it is not easy to make a critical comparison with other experimental results. We, however, refer to the result of the photoemission study (Schwentner et al 1981). They reported the diffusion length of the exciton in solid $\mathrm{Kr}$ of the range between 1 and $10 \mathrm{~nm}$, which is consistent with the present value.

3.3.2. Metastable atom desorption We also measured the absolute desorption yield of metastable atoms by exciton creation for $\mathrm{Ne}$ and $\mathrm{Ar}$ (Hirayama et al 1997). Experimental procedures to deduce the absolute yield are described in section 2.4.1. The results are listed in table 3. Only rough estimation for Ar (S1) is given because of unknown angular distribution for desorbed metastable Ar atoms and low signal intensity.

Comparison between the metastable and total desorption yield at S1 excitation shows that about $1 \%$ of the desorbed Ne atoms are in the metastable state $\left({ }^{3} \mathrm{P}_{0,2}\right)$, rest of which can be excited atoms in optically-allowed states $\left({ }^{1,3} \mathrm{P}_{1}\right)$.

\section{4. summary}

Our experimental results on the metastable and total desorption initiated by exciton creation were presented and the validity of three desorption mechanisms, cavity ejection (CE), excimer dissociation (ED) and internal sputtering (IS), was demonstrated. Observation of metastable atoms is found to be one of the most powerful tools for understanding the dynamic nature of excitons, especially on a surface, because an exciton created on the surface can desorb keeping its 'memory' as it was on the surface, such as its interaction with surrounding atoms, local geometrical condition, local temperature, etc. The results of the absolute desorption yields provided us a quantitative understanding of the exciton induced desorption processes.

It should be noted that some of important results are missing in this paper. The results for mixed (Hirayama et al 1996, Weibel et al 1996) and adsorbed (Weibel et al 1993) system can show another aspect of the exciton dynamics. In the viewpoint of vacuum technology, adsorption of residual gases on the cryogenic surface can be a serious problem. Very small amount of residual gas adsorption on rare gas solid surfaces was found to have a considerable influence on the desorption of ions (Sakurai et al 1990) and metastable atoms (Kuninobu et al 1997, Hayama et al 1998).

\section{Acknowledgments}

All of the data presented in this paper was obtained at UVSOR facility, Institute for Molecular Science, Okazaki, under the Joint Studies Program. Authors are grateful to the staff of the facility for their kind help throughout the work. We acknowledge a long-standing collaboration with Professor Makoto Sakurai, Kobe University. This 
work was partly supported by Grant-in-Aid for Scientific Research from the Ministry of Education, Science, Sports and Culture, Japan. 


\section{References}

Adachi T, Hirayama T, Miura T, Arakawa I \& Sakurai M 2003 Surf. Sci. 52860

Alvariño J M, Hepp C, Kreiensen M, Staudenmayer B, Vecchiocattivi F \& Kempter V 1984 J. Chem. Phys. $\mathbf{8 0} 765$

Arakawa I 1998 Molecular Crystals and Liquid Crystals Vol. 314 (New York: Gordon and Breach Science Publishers) p 47

Arakawa I, Adachi T, Hirayama T \& Sakurai M 2000 Surf. Sci. 451136

Arakawa I, Adachi T, Hirayama T \& Sakurai M 2003 Fizika Nizkikh Temperatur (Low Temperature Physics) 29259

Arakawa I, Takahasi M \& Takeuchi K 1989 J. Vac. Sci. Tech. A 72090

Arakawa I, Weibel D E, Nagai T, Abo M, Hirayama T, Kanno M, Mitsuke K \& Sakurai M 1995 Nucl. Instrum. Meth. Phys. Res. B 101195

Borst W 1971 Rev. Sci. Instrum. 421543

Buller W \& Johnson R 1991 Phys. Rev. B 436118

Chen L F, Huang G Q \& Song K S 1996 Nucl. Instrum. Meth. Phys. Res. B 11661

Cohen J S \& Schneider B 1974 J. Chem. Phys. 613230

Coletti F \& Debever J M 1984 J. Luminesc. 31/32 927

Coletti F, Debever J \& Zimmerer G 1984 J. Physique Lett. 45 L467

Cui S \& Johnson R 1989 Phys. Rev. B 399580

Cui S, Johnson R \& Cummings P 1988 Surf. Sci. 207186

Cui S, Johnson R E, Reimann C T \& Boring J W 1989 Phys. Rev. B 3912345

Dutkiewicz Ł, Pȩdrys R \& Schou J 1995 Nucl. Instrum. Meth. Phys. Res. B 101188

Dutkiewicz E, Pȩdrys R \& Schou J 1996 Europhys. Lett. 36301

Ellegaard O, Schou J \& Sørensen H 1986 Nucl. Instrum. Meth. Phys. Res. B 13567

Feulner P, Muller T, Puschmann A \& Menzel D 1987 Phys. Rev. Lett. 59791

Grigorashchenko O, Ogurtsov A, Savchenko E, Becker J, Runne M \& Zimmerer G 1997 Surf. Sci. 390277

Haensel R, Eitel G, Koch E E, Skibowski M \& Schreiber P 1970 Opt. Comm. 259

Hayama A, Kuninobu T, Hirayama T \& Arakawa I 1998 J. Vac. Sci. Tech. A 16979

Hirayama T, Hayama A, Adachi T, Arakawa I \& Sakurai M 2001 Phys. Rev. B 63075407

Hirayama T, Hayama A, Koike T, Kuninobu T, Arakawa I, Mitsuke K, Sakurai M \& Savchenko E V 1997 Surf. Sci. 390266

Hirayama T, Nagai T, Abo M, Arakawa I, Mitsuke K \& Sakurai M 1996 J. Electr. Spectr. Rel. Phen. 80101

Inoue K, Sakamoto H \& Kanzaki H 1984 J. Phys. Soc. Jpn. 53819

Johnson R \& Brown W 1982 Nucl. Instrum. Meth. Phys. Res. 198103

Johnson R \& Inokuti M 1983 Nucl. Instrum. Meth. Phys. Res. 206289

Kato H, Furuhashi O \& Hirayama T 2006 in preparation .

Kloiber T, Laasch W, Zimmerer G, Coletti F \& Debever J M 1988 Europhys. Lett. 777

Kloiber T \& Zimmerer G 1989 Radiat. Eff. Def. Solids 109219

Kloiber T \& Zimmerer G 1990 Physica Scripta 41962

Kuninobu T, Hayama A, Hirayama T \& Arakawa I 1997 Surf. Sci. 390272

Leclerc G, Bass A D, Mann A \& Sanche L 1992 Phys. Rev. B 464865

Leclerc G, Bass A D, Michaud M \& Sanche L 1990 J. Elect. Spectr. Relat. Phenom. 52725

Pudewill D, Himpsel F J, Saile V, Schwentner N, Skibowski M \& Koch E E 1976 Phys. Stat. Sol. B 74485

Reimann C T, Brown W L, Grosjean D E \& Nowakowski M J 1992 Phys. Rev. B 4543

Reimann C T, Brown W L \& Johnson R E 1988 Phys. Rev. B 37(4), 1455

Reimann C T, Brown W L, Nowakowski M J, Cui S \& Johnson R E 1990 Desorption Induced by Electronic Transitions, DIET-IV ed G Betz \& P Varga (Heidelberg: Springer) p. 226

Reimann C T, Johnson R E \& Brown W L 1984 Phys. Rev. Lett. 53(6), 600

Runne M, Becker J, Laasch W, Varding D \& Zimmerer G 1993 Nucl. Instrum. Meth. Phys. Res. B 82301

Saile V \& Koch E E 1979 Phys. Rev. B 20784

Sakurai M, Adachi T, Hirayama T \& Arakawa I 2002 UVSOR Activity Report, UVSOR-29 p. 214

Sakurai M, Hirayama T \& Arakawa I 1990 Vacuum 41217

Sakurai M, Morita S, Fujita J, Yonezu H, Fukui K, Sakai K, Nakamura E, Watanabe M, Ishiguro E \& Yamashita K 1989 Rev. Sci. Instrum. 602089

Sakurai M, Nagai T, Abo M, Hirayama T \& Arakawa I 1995 J. Vac. Soc. Jpn. 38298

Saloman E B \& Sansonetti C J 2004 J. Phys. Chem. Ref. Data 331113 
Samson J A R 1964 J. Opt. Soc. Am. 546

Savchenko E V, Hirayama T, Hayama A, Koike T, Kuninobu T, Arakawa I, Mitsuke K \& Sakurai M 1997 Surf. Sci. 390261

Schou J 1987 Nucl. Instrum. Meth. Phys. Res. B $\mathbf{2 7} 188$

Schwentner N, Himpsel F J, Saile V, Skibowski M, Steinmann W \& Koch E E 1975 Phys. Rev. Lett. 34528

Schwentner N \& Koch E E 1976 Phys. Rev. B 144687

Schwentner N, Martens G \& Rudolf H W 1981 Phys. Stat. Sol. B 106183

Skibowski M 1971 Proc. 3rd Int. Conf. on VUV Rad. Phys. (Tokyo)

Small-Warren N E \& Chiu L Y C 1975 Phys. Rev. A 111777

Sonntag B 1977 Rare Gas Solids Vol. 2 ed M L Klein \& J A Venables (London: Academic Press) p 1022

Weibel D E, Hirayama T \& Arakawa I 1993 Surf. Sci. 283204

Weibel D E, Hoshino A, Hirayama T, Sakurai M \& Arakawa I 1993 Desorption Induced by Electronic Transitions - V ed A R Burns, E B Stechel \& D R Jenninson (Springer-Verlag) p 333

Weibel D E, Nagai T, Hirayama T, Arakawa I \& Sakurai M 1996 Langmuir 12193

Zimmerer G 1994 Nucl. Instrum. Meth. Phys. Res. B 91601 
Table 1. Kinetic energies and electronic states of desorbed metastable Ne atoms.

\begin{tabular}{llllll}
\hline & $\begin{array}{l}\text { Electronic } \\
\text { Configuration }\end{array}$ & $\begin{array}{l}\text { Excitation } \\
\text { Energy }(\mathrm{eV})\end{array}$ & $\begin{array}{l}\text { Kinetic } \\
\text { Energy }(\mathrm{eV})\end{array}$ & $\begin{array}{l}\text { Desorption } \\
\text { Mechanism }\end{array}$ & $\begin{array}{l}\text { Electronic State } \\
\text { at Desorption }\end{array}$ \\
\hline S1 & $2 \mathrm{p}^{5} 3 \mathrm{~s}$ & 17.2 & 0.18 & $\mathrm{CE}$ & $2 \mathrm{p}^{5} 3 \mathrm{~s}\left({ }^{3} \mathrm{P}_{0,2}\right)$ \\
$\mathrm{B} 1$ & $2 \mathrm{p}^{5} 3 \mathrm{~s}$ & 17.5 & 0.18 & $\mathrm{CE}$ & $2 \mathrm{p}^{5} 3 \mathrm{~s}\left({ }^{3} \mathrm{P}_{0,2}\right)$ \\
& & & $\leq 0.05$ & $\mathrm{IS}$ & $2 \mathrm{p}^{5} 3 \mathrm{~s}\left({ }^{3} \mathrm{P}_{0,2}\right)$ \\
$\mathrm{S}$ & $2 \mathrm{p}^{5} 3 \mathrm{p}$ & \multirow{2}{*}{19.0} & 0.19 & $\mathrm{CE}(\mathrm{bulk})$ & $2 \mathrm{p}^{5} 3 \mathrm{p}$ or $2 \mathrm{p}^{5} 3 \mathrm{~s}$ \\
& & $0.2 \sim 0.4$ & $\mathrm{CE}$ & $2 \mathrm{p}^{5} 3 \mathrm{p}$ \\
& & 1.4 & $\mathrm{ED}$ & \\
$\mathrm{B} 2$ & $2 \mathrm{p}^{5} 4 \mathrm{~s}$ & \multirow{2}{*}{20.3} & 0.18 & $\mathrm{CE}$ & $2 \mathrm{p}^{5} 3 \mathrm{~s}\left({ }^{3} \mathrm{P}_{0,2}\right)$ \\
& & 0.36 & $\mathrm{CE}$ & $2 \mathrm{p}^{5} 4 \mathrm{~s}$ \\
& & 1.4 & $\mathrm{ED}$ & \\
& & $\leq 0.05$ & $\mathrm{IS}$ & $2 \mathrm{p}^{5} 3 \mathrm{~s}\left({ }^{3} \mathrm{P}_{0,2}\right)$ \\
\hline
\end{tabular}

Table 2. Fitting parameter $n$ and FWHM of angular distribution of desorbed metastable atoms from the surface of solid Ne.

\begin{tabular}{cccc}
\hline Exciton & Mechanism & $n$ & FWHM (deg.) \\
\hline S1 & CE & 14 & 36 \\
B1 & CE & 10 & 42 \\
S' & CE & 8 & 47 \\
& ED & $0 \sim 1$ & $\geq 120$ \\
B1 tail & IS & $2 \sim 4$ & $70 \sim 90$ \\
\hline
\end{tabular}


Table 3. Absolute yields of the total and metastable desorption from solid Ne, Ar, and $\mathrm{Kr}$.

\begin{tabular}{|c|c|c|c|c|}
\hline & Exciton & Desorbed Species & $\begin{array}{l}\text { Desorption Yield } \\
\text { (atoms/photon) }\end{array}$ & Ref. \\
\hline \multirow[t]{8}{*}{$\mathrm{Ne}$} & $\mathrm{S} 1$ & Total & 0.3 & $\mathrm{~b}$ \\
\hline & & Metastable & $(2.3 \pm 0.7) \times 10^{-3}$ & $\mathrm{c}$ \\
\hline & $\mathrm{S}^{\prime}$ & Total & 0.1 & $\mathrm{~b}$ \\
\hline & & Metastable & $(7.8 \pm 2.3) \times 10^{-4}$ & $\mathrm{c}$ \\
\hline & B1 & Total & $1.6 \pm 0.3$ & $\mathrm{~b}$ \\
\hline & & Metastable & $(1.4 \pm 0.4) \times 10^{-3}$ & $\mathrm{c}$ \\
\hline & $\mathrm{B} 2$ & Total & $1 \pm 0.2$ & $\mathrm{~b}$ \\
\hline & & Metastable & $1 \times 10^{-3}$ & $\mathrm{c}$ \\
\hline \multirow[t]{5}{*}{ Ar } & $\mathrm{S} 1$ & Total & 0.1 & $\mathrm{~d}$ \\
\hline & & Metastable $^{a}$ & $1 \times 10^{-5}$ & $\mathrm{c}$ \\
\hline & $\mathrm{S} 2$ & Total & 0.1 & $\mathrm{~d}$ \\
\hline & B1 & Total & 0.23 & $\mathrm{~d}$ \\
\hline & $\mathrm{B} 2$ & Total & 0.16 & d \\
\hline \multirow[t]{6}{*}{$\mathrm{Kr}$} & $\mathrm{S} 1(3 / 2)$ & Total & 0.015 & e \\
\hline & $\mathrm{S} 1(1 / 2)$ & Total & 0.01 & $\mathrm{e}$ \\
\hline & $\mathrm{S} 2(3 / 2)$ & Total & $\leq 10^{-3}$ & $\mathrm{e}$ \\
\hline & $\mathrm{B} 1(3 / 2)$ & Total & 0.03 & $\mathrm{e}$ \\
\hline & $\mathrm{B} 1(1 / 2)$ & Total & 0.02 & $\mathrm{e}$ \\
\hline & $\mathrm{B} 2(3 / 2)$ & Total & $\leq 10^{-3}$ & e \\
\hline
\end{tabular}

a only rough estimation.

b From Arakawa et al (2000).

c From Hirayama et al (1997).

d From Arakawa et al (2003).

e From Adachi et al (2003). 


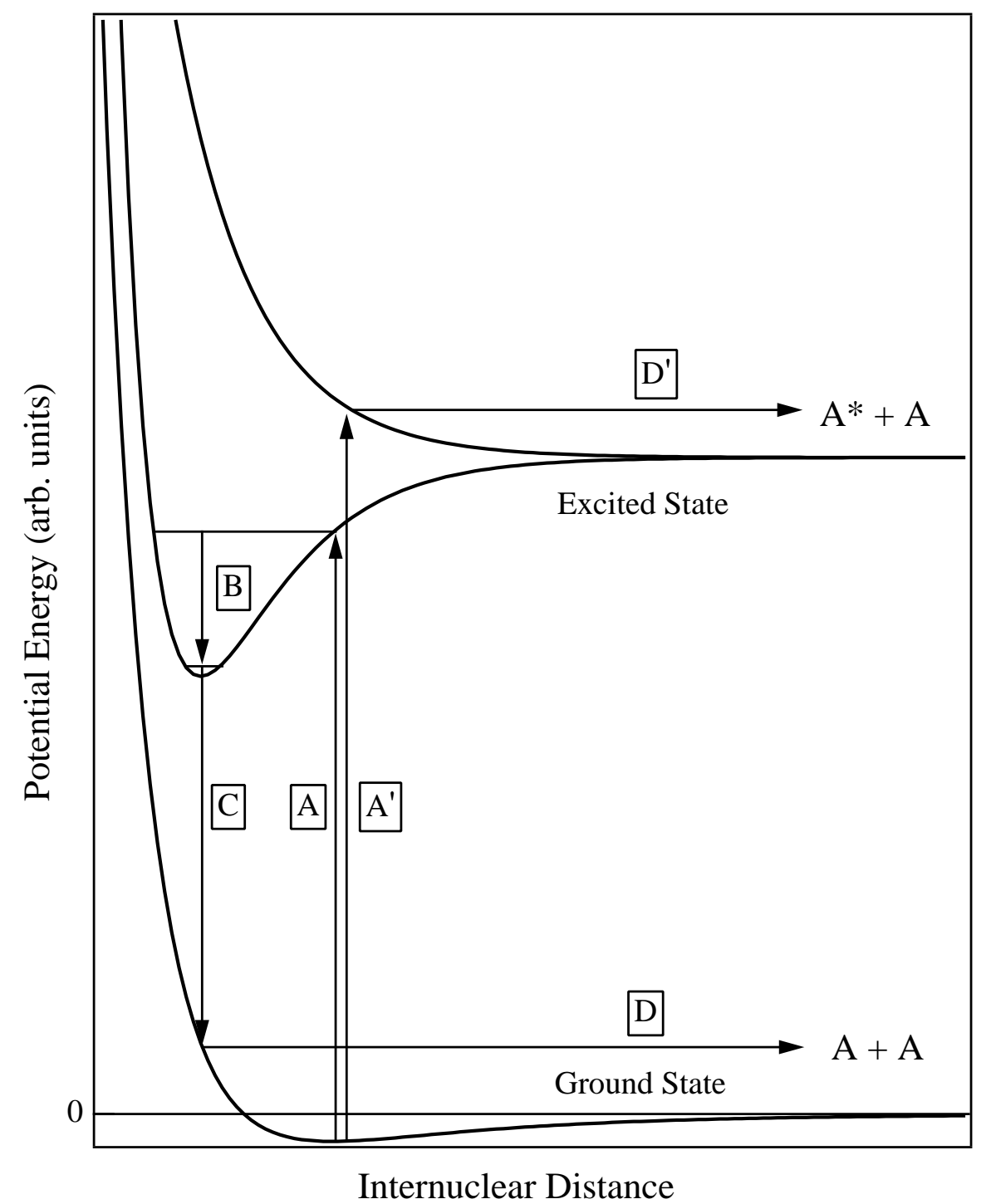

Figure 1. Schematic potential curve for the excimer dissociation mechanism. See text for details. 
Photodesorption in rare gas solids

16

Exciton creation on the surface

Cavity formation
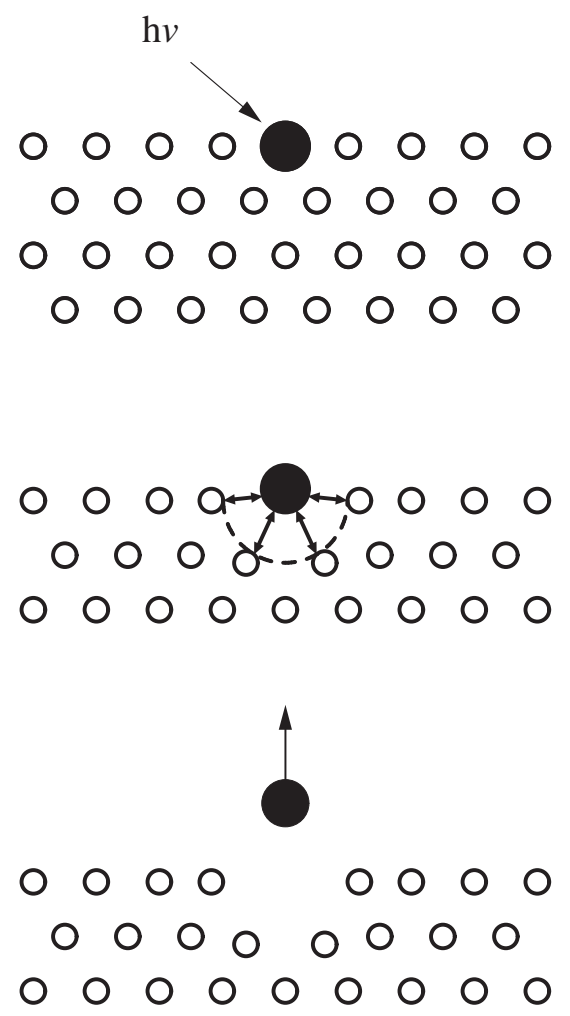

Figure 2. Schematic of the cavity ejection mechanism. Solid and open circles represent the excited and ground state atoms, respectively. See text for details. 


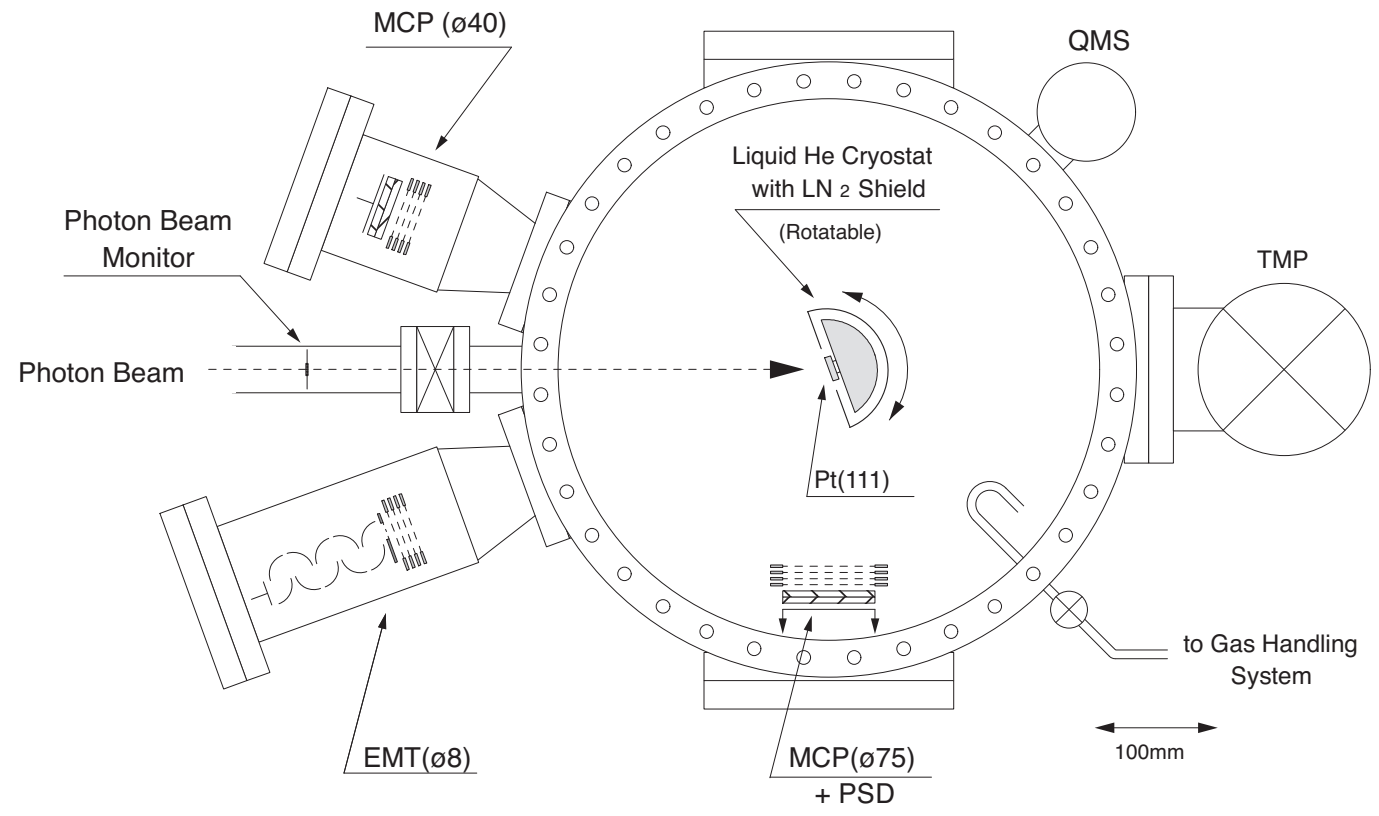

Figure 3. Schematic view of the main chamber. 


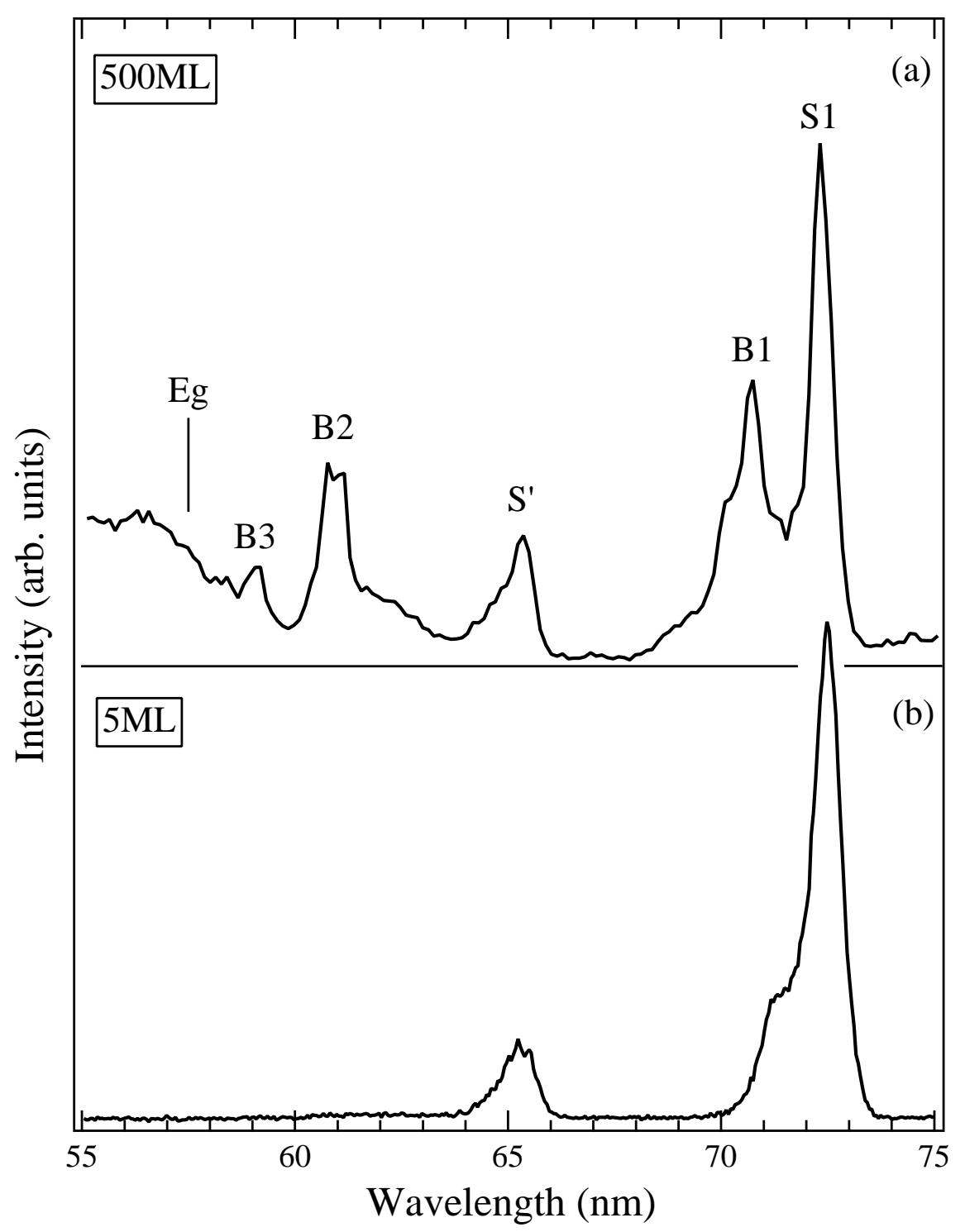

Figure 4. Dependence of metastable $\mathrm{Ne}^{*}$ yield via the cavity ejection mechanism from the surface of solid Ne on the wavelength of excitation light. The assignments for each peaks and the position of the band gap energy $E_{\mathrm{g}}$ are shown. The thickness of the sample is (a) 500 and (b) 5 atomic monolayers. From Hirayama et al (1997). 


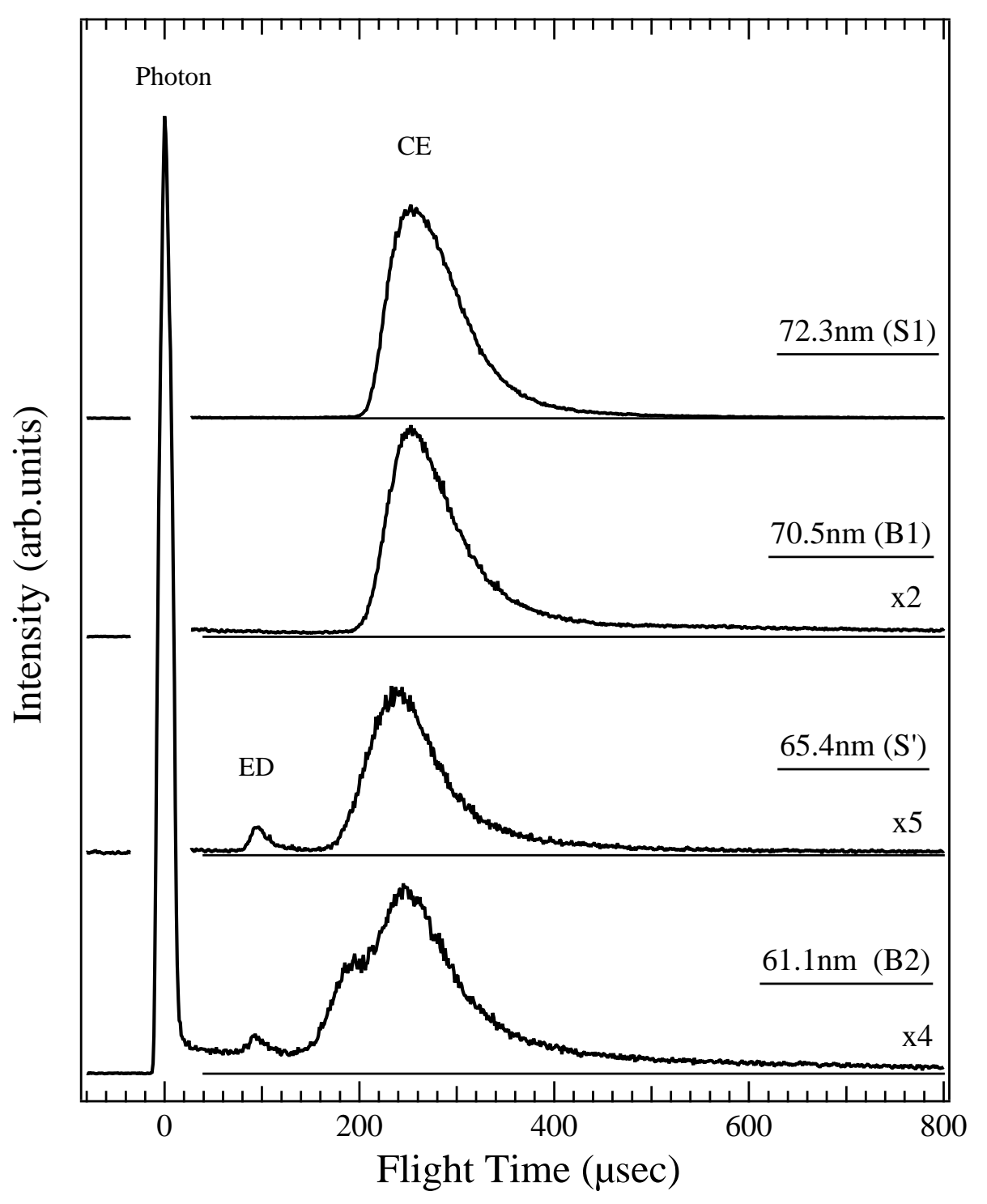

Figure 5. Time-of-flight spectra of desorbed metastable Ne atoms from solid $\mathrm{Ne}$ at excitation wavelengths corresponding to the surface (S1, S') and bulk (B1, B2) excitons. From Hirayama et al (1996). 


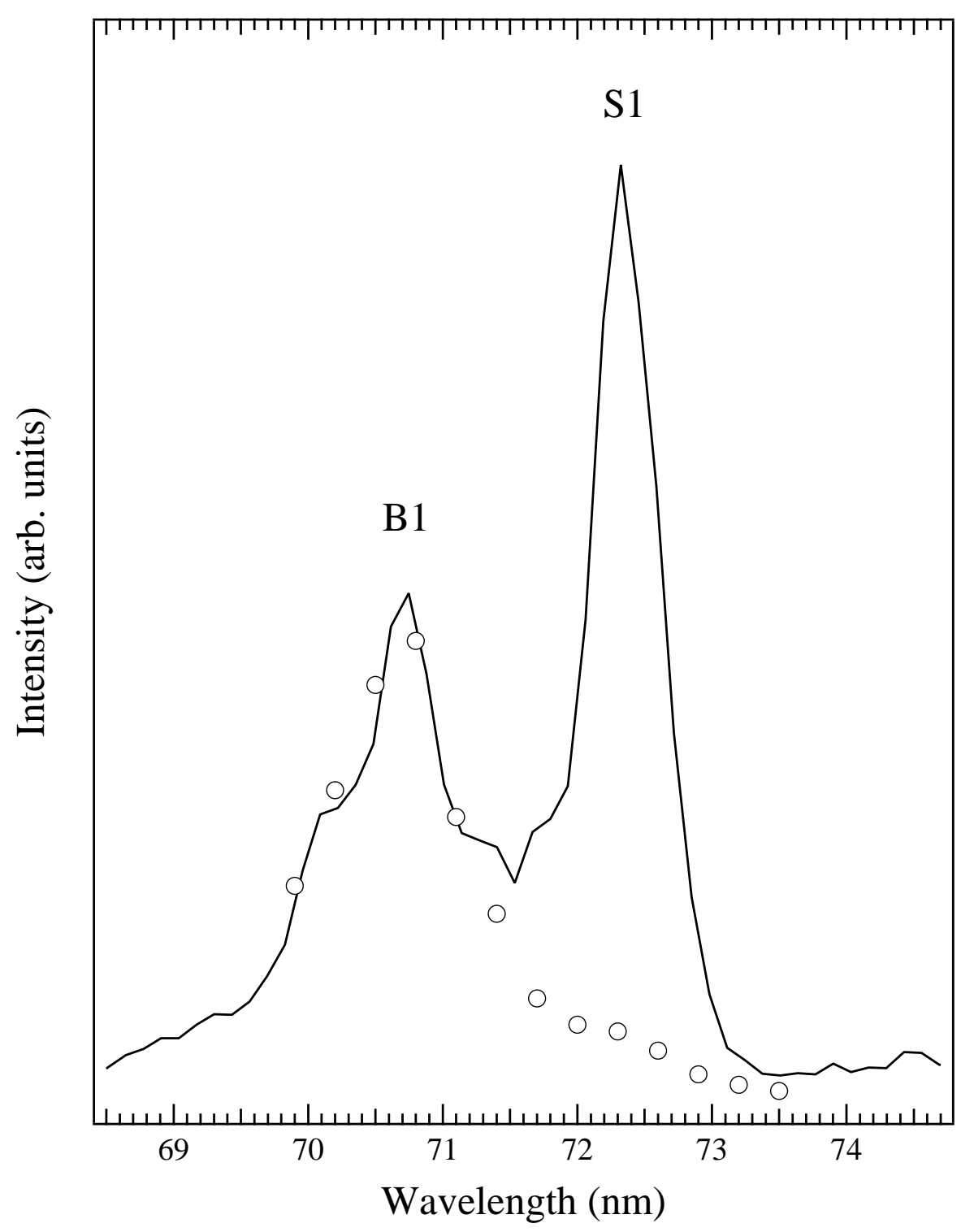

Figure 6. Wavelength dependence of the CE intensity (solid line) and the area of the tail in the TOF spectra (open circles). The area of the tail was taken at the region of $t_{\mathrm{f}}=600 \sim 900 \mu \mathrm{s}$, which correspond to the kinetic energy $E_{\mathrm{k}}: 0.01 \sim 0.03 \mathrm{eV}$. The scale is arbitrary in each curve. 


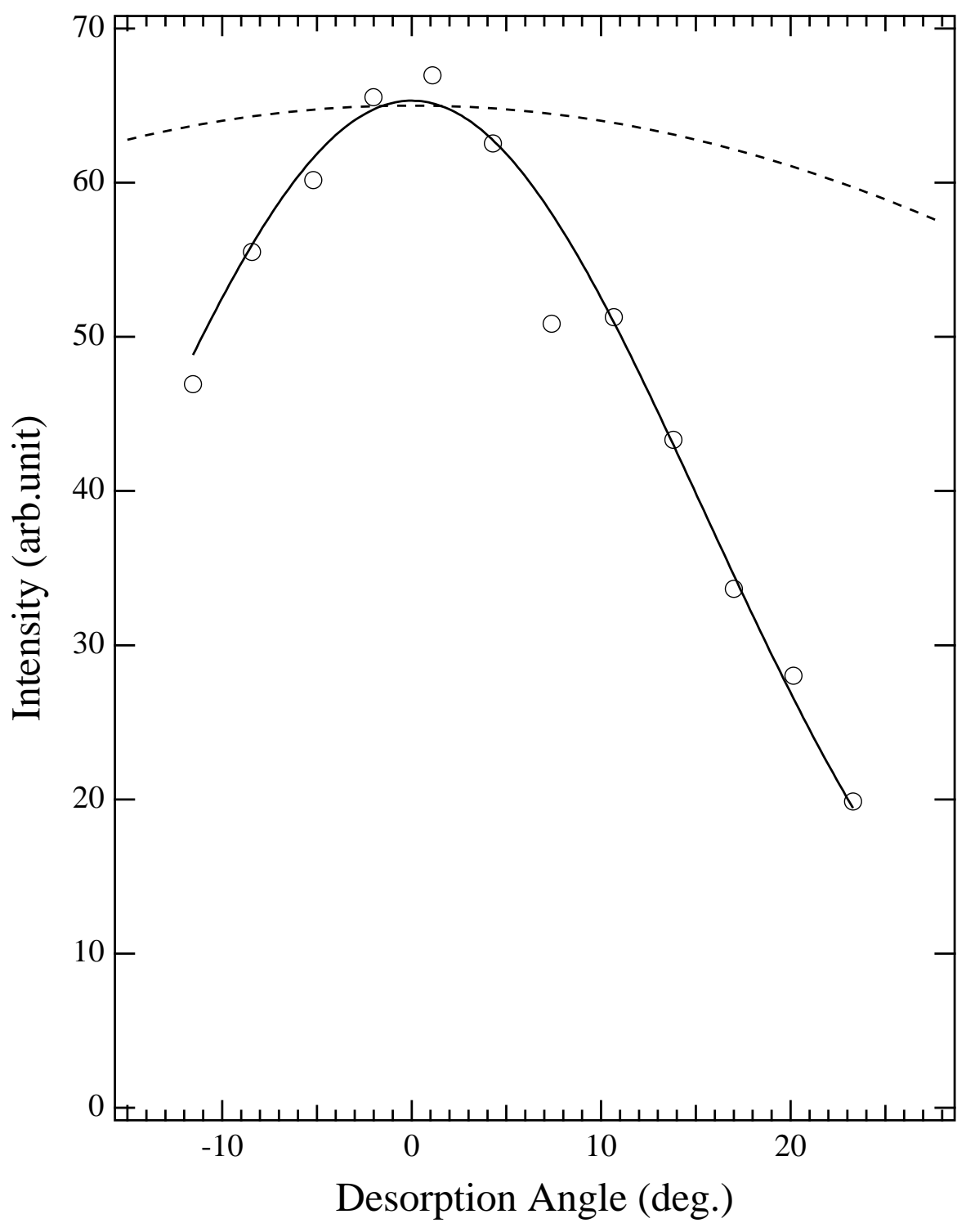

Figure 7. Angular distribution of the desorbed metastable Ne atoms via the cavity ejection mechanism by the excitation of the first order surface exciton (S1). Solid line is the fitting curve using $\cos ^{n} \theta$ where $n=14$. Dashed line shows $\cos \theta$ for comparison. From Arakawa et al (1995). 


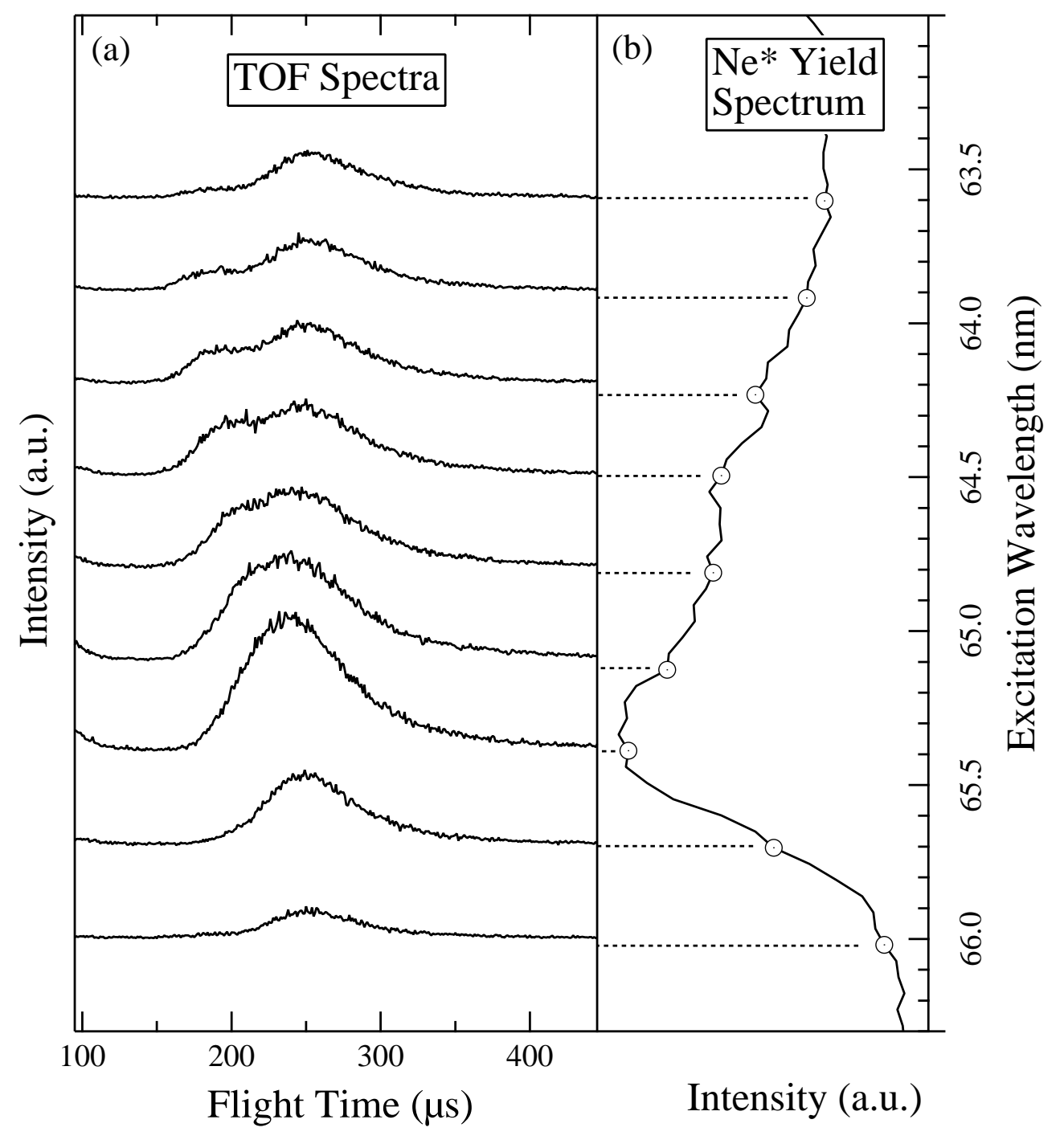

Figure 8. (a) Series of time-of-flight spectra and (b) dependence of $\mathrm{Ne}^{*}(\mathrm{CE})$ intensity on the wavelength of incident light in the wavelength range of $63-65.7$ $\mathrm{nm}$ corresponding the excitation energy of S' exciton. Thickness of the sample was $690 \mathrm{ML}$. The wavelengths where each TOF spectrum in (a) was measured are marked by open circles in (b). 


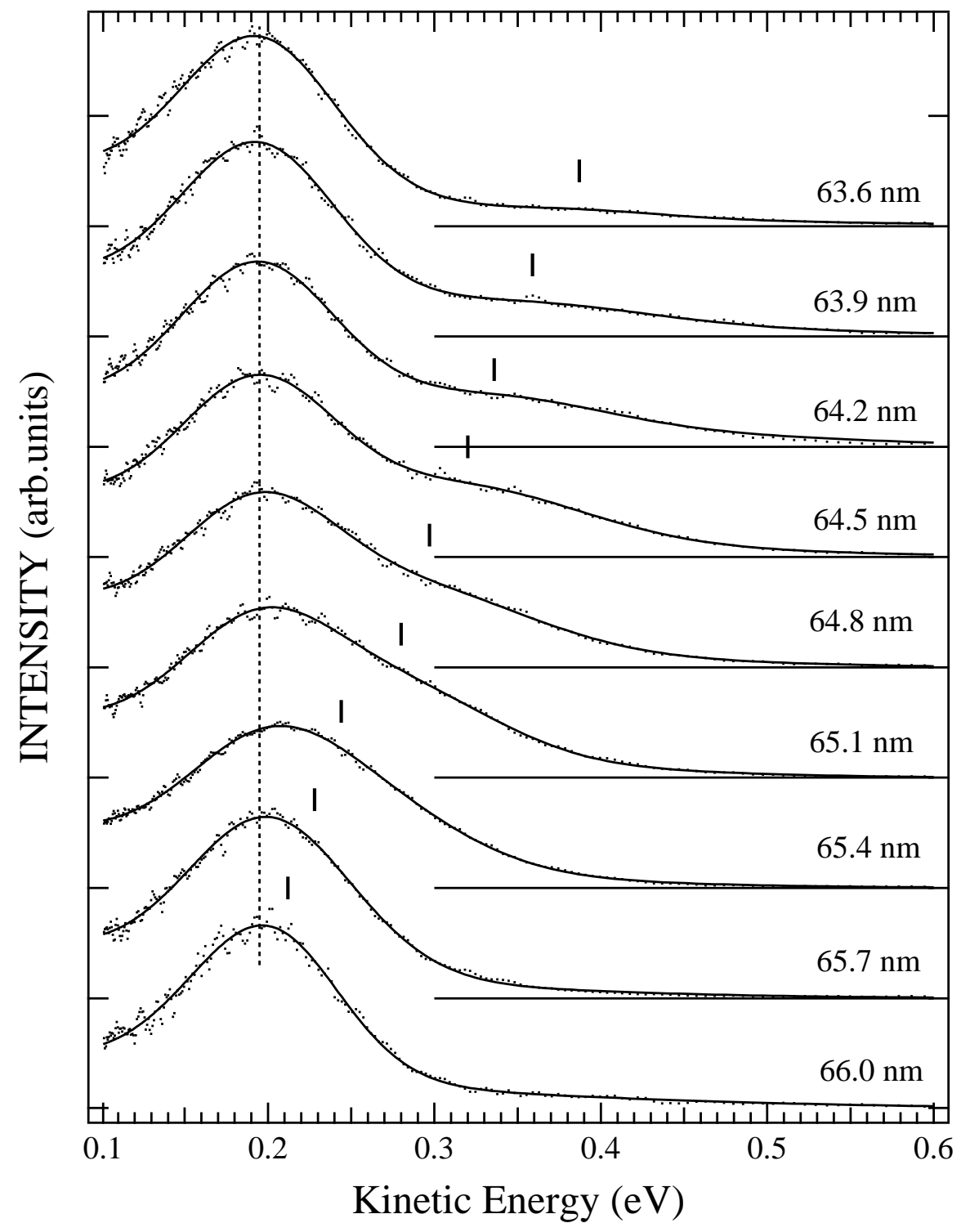

Figure 9. Kinetic energy spectra of desorbed $\mathrm{Ne}^{*}(\mathrm{CE})$ by the excitation of S' excitons. Dots and solid lines are measured fitted results. Vertical solid lines and dashed line are the peak positions of the peak (A) and peak (B), respectively. 


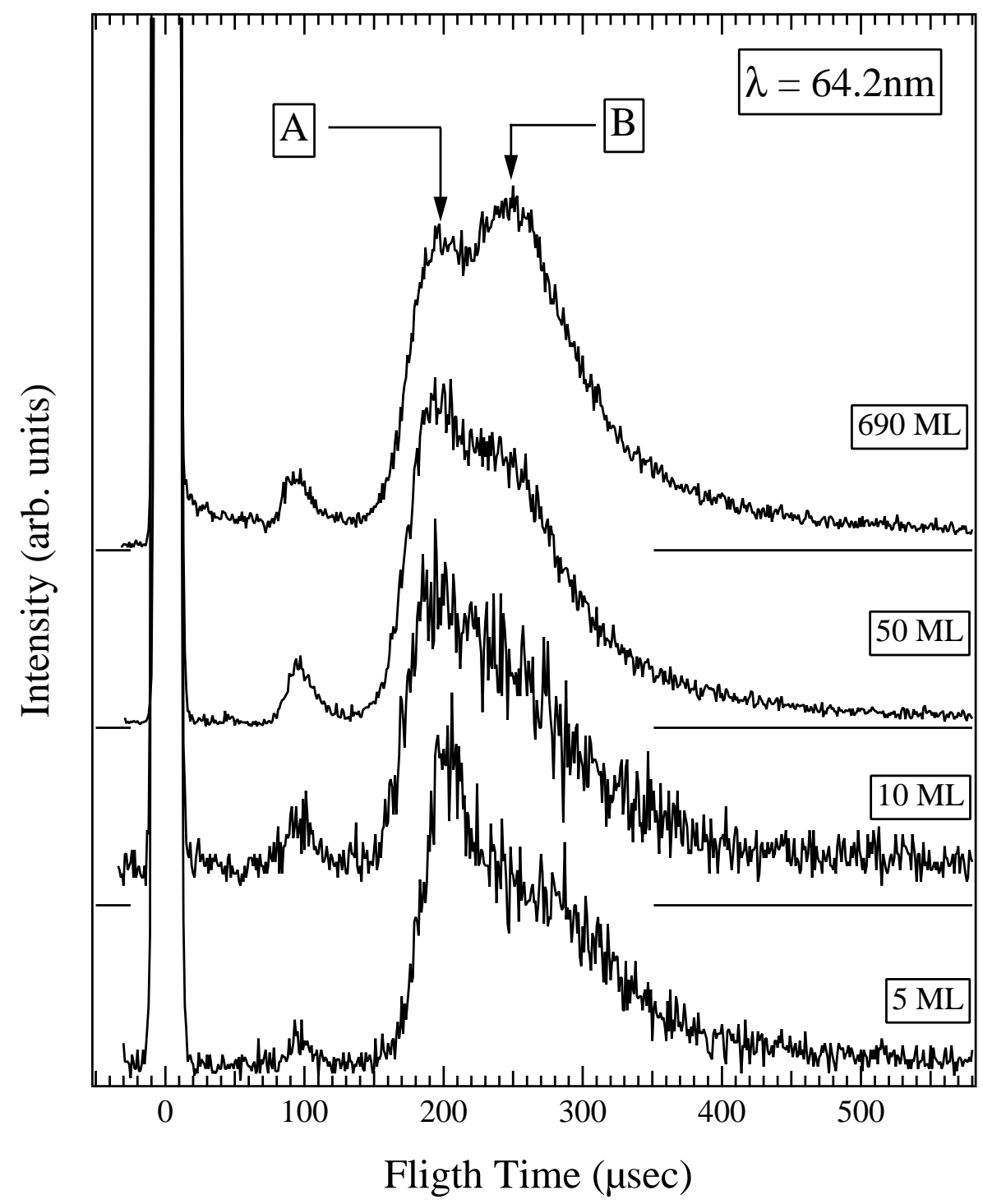

Figure 10. Thickness dependence of the time-of-flight spectra of $\mathrm{Ne}^{*}$ measured at the wavelength of $64.2 \mathrm{~nm}$. 


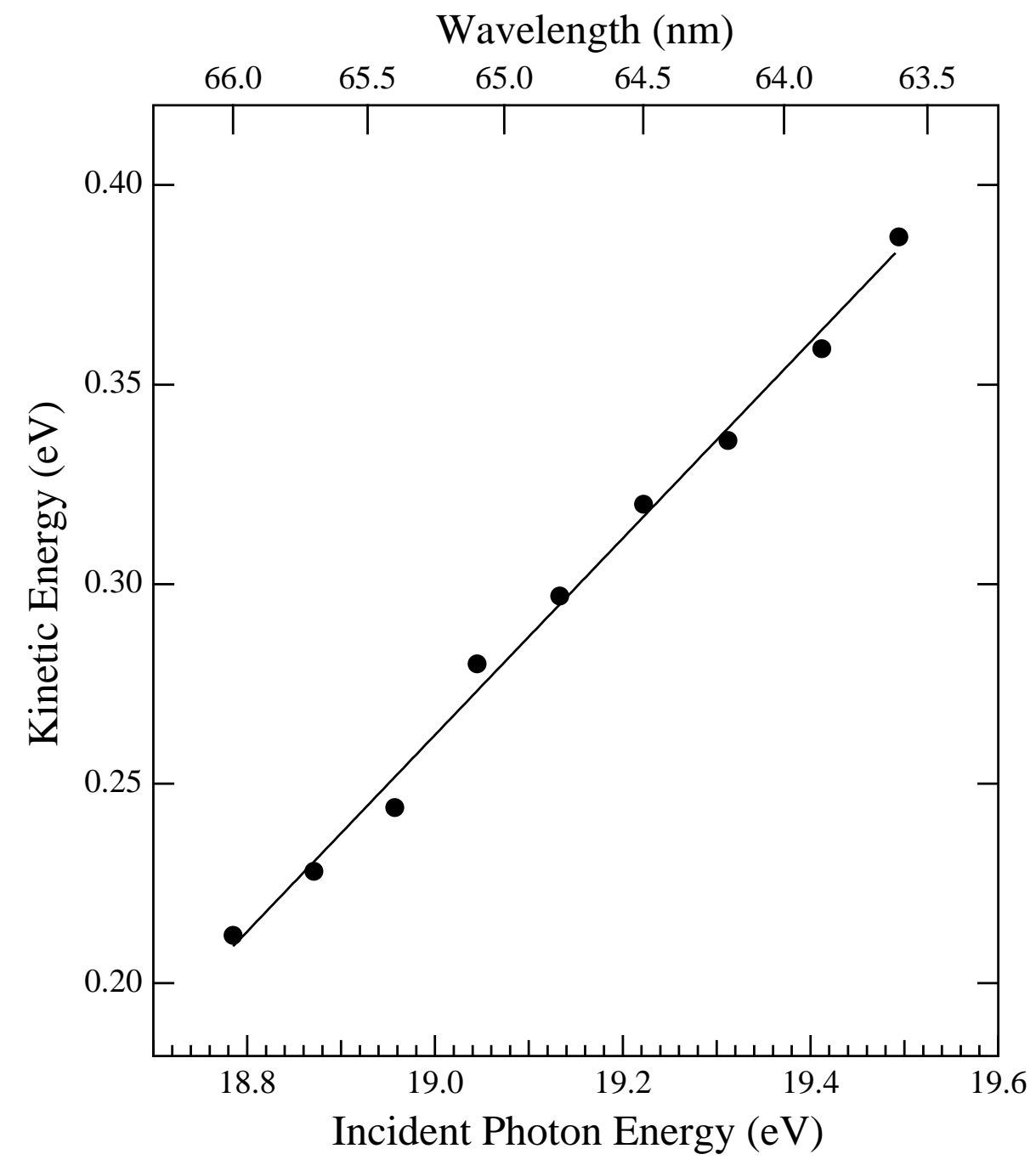

Figure 11. Kinetic energy of peak (A) as a function of the incident photon energy. Solid line is a guide to the eye. 


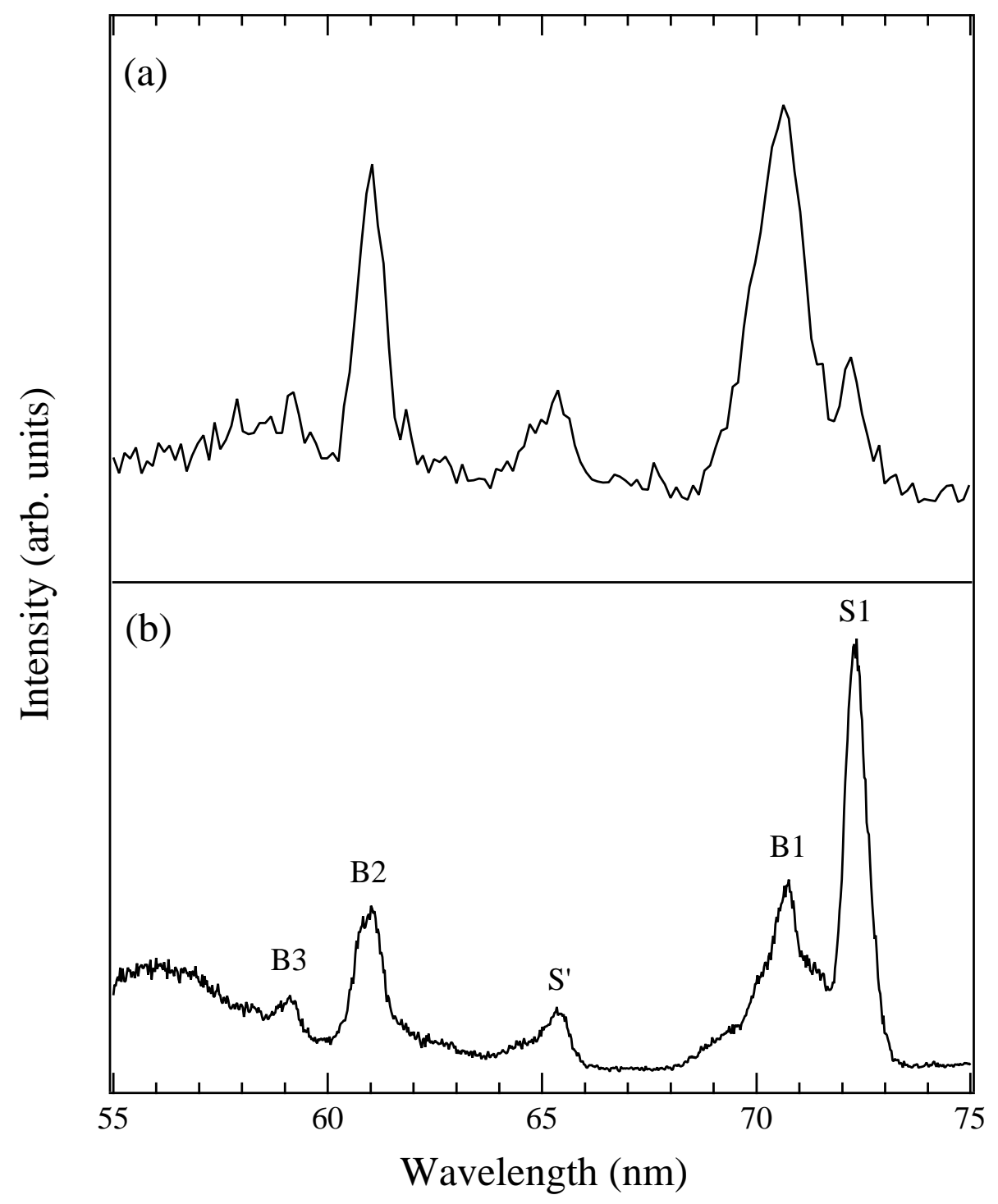

Figure 12. (a) Plume emission intensity, or the desorption yield of $\mathrm{Ne}_{2} *\left({ }^{3} \Sigma_{u}\right)$ from the surface of solid Ne vs. the wavelength of stimulating light. (b) Yield of excited $\mathrm{Ne}$ atoms $\mathrm{Ne}^{*}$ in ${ }^{3} \mathrm{P}_{0,2}$ states that are desorbed from the surface of solid Ne through the cavity ejection mechanism. From Hirayama et al (2001). 


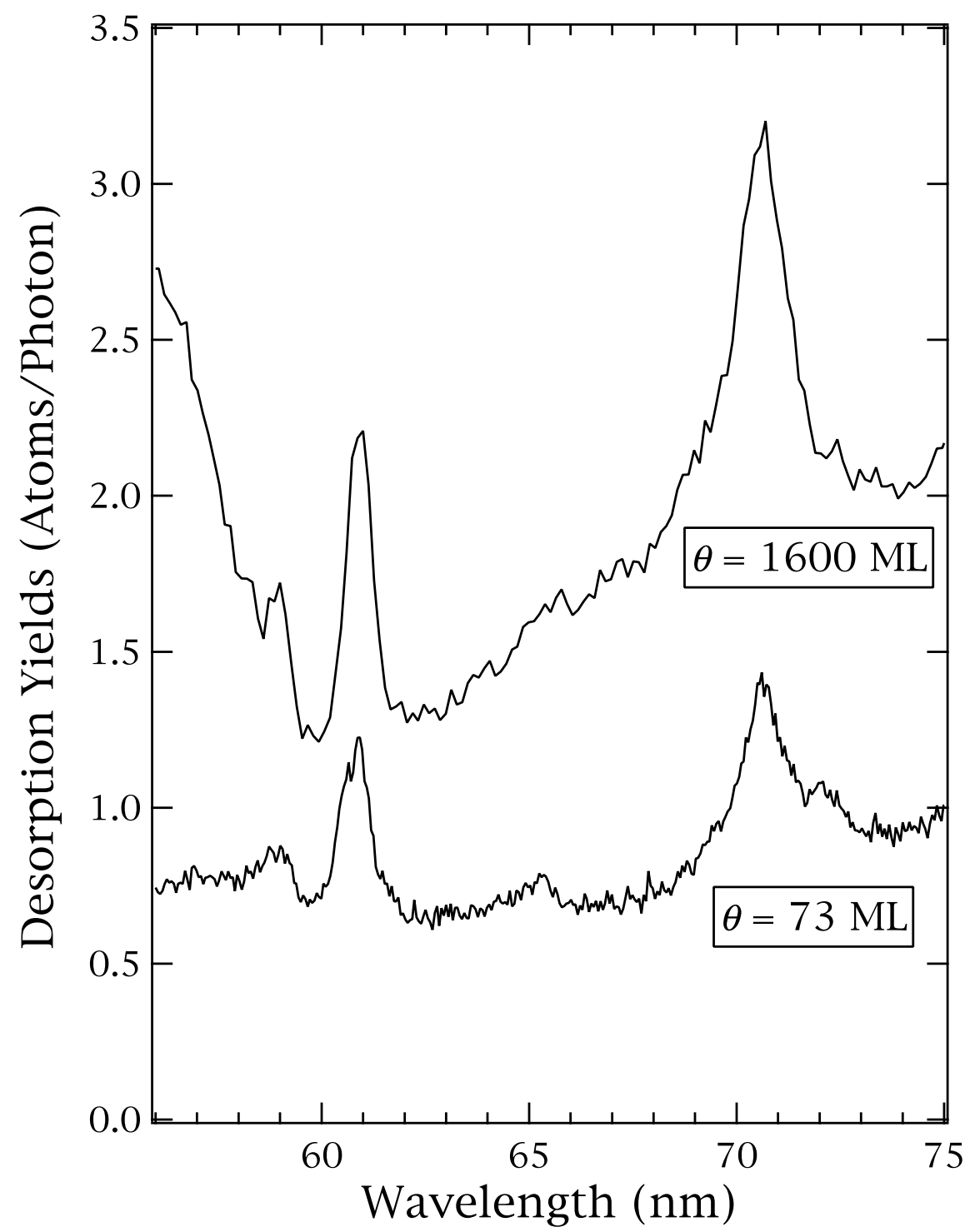

Figure 13. Absolute desorption yield spectra for solid $\mathrm{Ne}$ in the excitonic excitation range, $56-75 \mathrm{~nm}$ of wavelength. The thickness of the sample was 1600 and 73 atomic layers. From Arakawa et al (2000). 


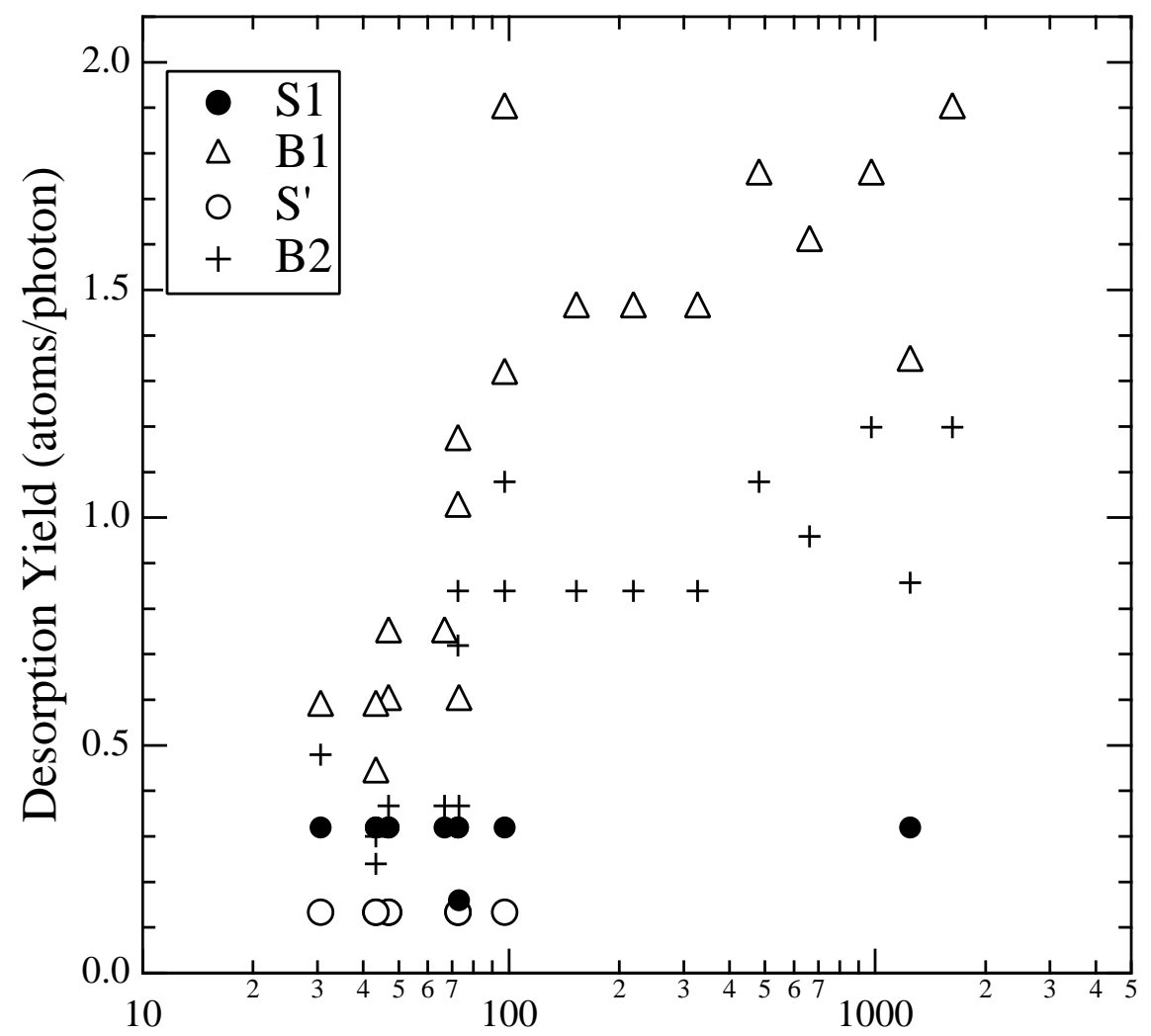

Film Thickness (atomic layers)

Figure 14. Thickness dependence of the desorption yields for solid $\mathrm{Ne}$ at the excitation of excitons, S1, B1, S' and B2. From Arakawa et al (2000). 


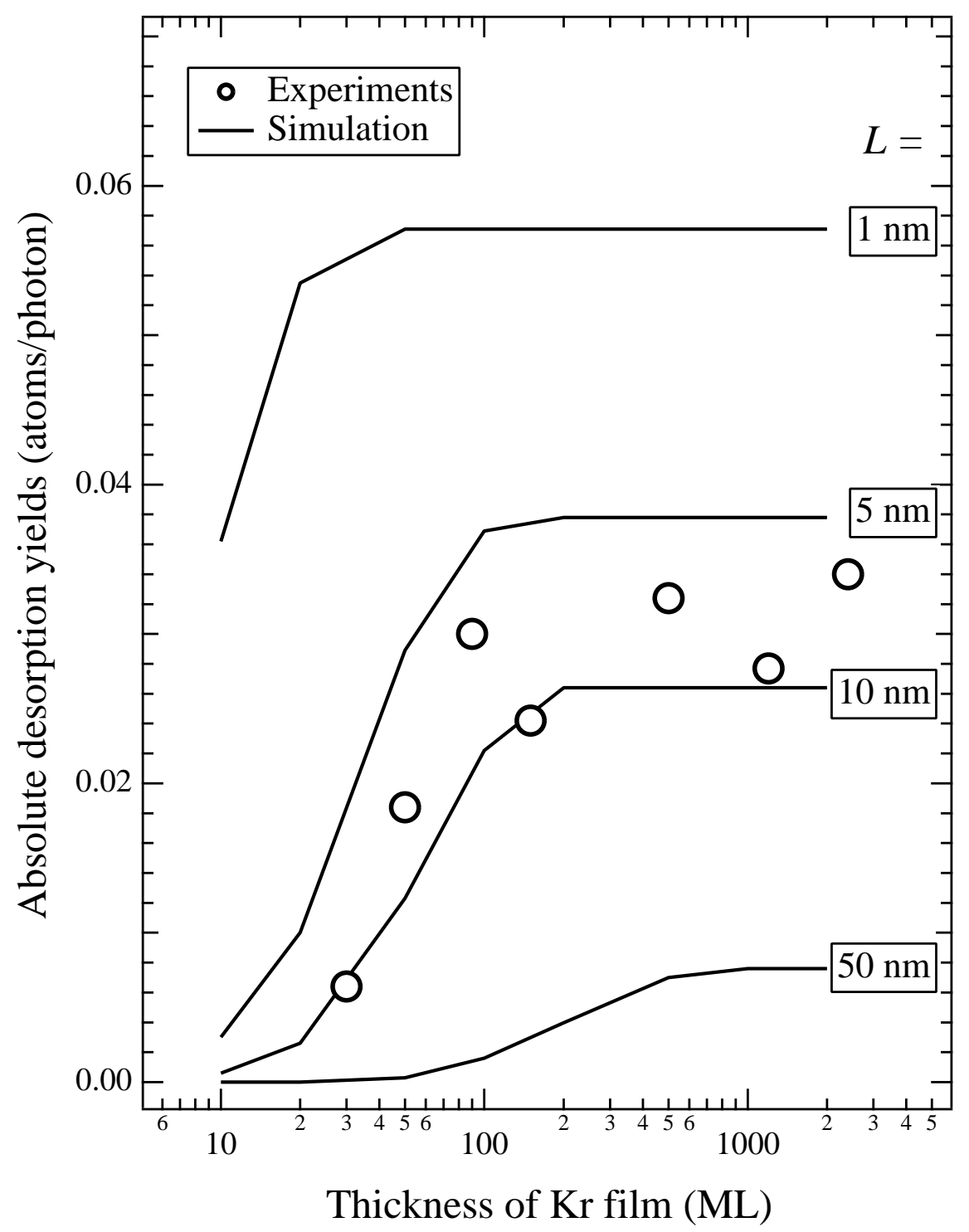

Figure 15. Thickness dependence of the desorption yields for solid $\mathrm{Kr}$ at the excitation of B1(3/2) excitons. Open circles: experimental results. Solid lines: simulation results. Diffusion length $L$ used for the calculation of each curve is also shown. See text for details. From Adachi et al (2003). 